\title{
Fachadas ventiladas activas para reducir la demanda de calefacción en los edificios de oficinas. El caso de España
}

\author{
Ventilated active façades to reduce heating demand \\ in office buildings. The case of Spain
}

O. Irulegi(*), A. Serra(*), R. Hernández ${ }^{(*)}$, A. Ruiz-Pardo(**), L. Torres ${ }^{(*)}$

RESUMEN

En este artículo se analiza la eficiencia energética de una Fachada Ventilada Activa -FVA- aplicada a edificios de oficinas en España para reducir la demanda de calefacción.

El sistema de fachada consiste en una hoja exterior de $2 \mathrm{~mm}$ de acero galvanizado y una cavidad de $3 \mathrm{~cm}$ donde el aire de ventilación es precalentado en invierno y extraído en verano.

Tras definir 8 tipologías típicas, se han obtenido 192 casos de estudio en los que se consideran diferentes parámetros: la superficie acristalada, la orientación y la zona climática.

El cálculo de la demanda energética de los casos de estudio ha sido llevado a cabo mediante el programa LIDER, el método general del Código Técnico de la Edificación - CTE. Los resultados obtenidos en términos de reducción de la demanda de calefacción, se muestran comparados con los mínimos requerimientos exigidos en el CTE.

$113-121$

Palabras clave: Eficiencia energética; estrategias de calefacción; fachada ventilada activa; oficinas.

\section{SUMMARY}

This paper analyses the energy efficiency of a Ventilated Active Façade -VAFapplied to office buildings in Spain to reduce heating demand.

This façade system consists of an outer layer element of $2 \mathrm{~mm}$ galvanized steel panels and a $3 \mathrm{~cm}$ air cavity where the ventilation air is preheated in winter and exhausted in summer.

After defining 8 typical office typologies in Spain, 192 study cases are obtained where different parameters like the percentage of glass in façade, the orientation and the climatic zone of the buildings are considered.

The energy demand of all these study cases is obtained using the official simulation tool of the Spanish Technical Building Regulation - CTE called LIDER. The results, in terms of reduction of heating demand, are presented in comparison with the minimum energetic requirements of CTE.

Keywords: Energy efficiency; heating strategies; ventilated active façade; office buildings.

\footnotetext{
(*) ETSA, Universidad del País Vasco. San Sebastián (España).

(**) Escuela Superior de Ingenieros, Universidad de Sevilla (España).

Persona de contacto/Corresponding author: o.irulegi@ehu.es (O. Irulegi)
} 
1. Funcionamiento de la FVA en modo invierno y verano y test del primer prototipo

\section{INTRODUCCIÓN}

Este artículo es parte de una tesis doctoral vinculada al proyecto de investigación denominado "Fachadas Ventiladas Activas" y que ha sido financiado por el Gobierno de España. El objetivo principal del proyecto era desarrollar un elemento de fachada opaco capaz de gestionar los flujos energéticos entre el interior y el exterior de un edificio. El proyecto de investigación finalizó en diciembre de 2009 y fue realizado de manera coordinada entre la Universidad del País Vasco, la Universidad de Sevilla y la Universidad de Córdoba.

La tesis doctoral (1), de la que se extrae este artículo, analiza la demanda energética de calefacción y refrigeración en edificios de oficinas dotados con tecnología FVA. En el presente artículo se presentan los resultados obtenidos para calefacción.

La FVA es un tipo de fachada de doble piel activa (Active Double Skin Façade). Según Arons et al (2): "Una fachada de doble piel (Double Skin Façade) se compone de dos capas que permiten el movimiento de aire exterior o interior en él. En algunas ocasionas también se hace referencia a las dobles pieles (twin skins)". Sin embargo, para otros autores (3) (4) (5), una fachada de doble piel (Double Skin Façade) está constituida necesariamente por superficies transparentes. En general, apenas se hace mención a superficies opacas.

Según el Belgian Building Research Institute $B B R I$ (6) "Una fachada activa es una fachada que cubre uno o varios pisos de un edificio y está construida por múltiples capas acristaladas. Las diferentes pieles pueden ser o no estancas. En este tipo de fachadas, la cavidad de aire situada entre las diferentes superficies, se ventila de manera mecánica o natural. La estrategia de ventilación de la cámara puede variar con el tiempo. Habitualmente se integran sistemas pasivos o activos que mejoran la calidad del aire en el interior del edificio. En la mayoría de los casos este tipo de sistemas suelen estar gestionados mediante mecanismos de control semi-automáticos".

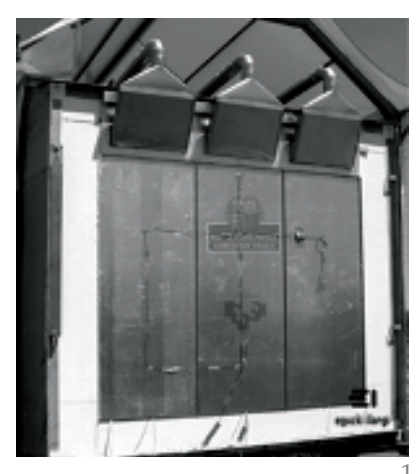

apertura de ventanas en primavera y otoño (cuando las temperaturas exteriores son más bajas)

La idea de construir un elemento exterior de fachada que gestione el flujo de calor, la humedad y el aire ha sido desarrollada en el Solar Dynamic Buffer Zone (SDBZ): "el concepto de Zona de Almacenamiento Dinámico - ZAD - (Dynamic Buffer Zone) contempla la ventilación de una cámara situada en el interior de un muro para controlar el movimiento de calor y de migración de humedad a través de la fachada, [..] la ZAD introduce aire caliente del exterior con un poco de humedad en la cavidad intersticial en la fachada". La calificación de "solar" fue añadida para especificar la manipulación de la transmisión de calor en la cavidad de la fachada.

Ya existen en el mercado sistemas que precalienten el aire de ventilación como el SolarWal/ $\mathbb{R}$ producido para el Departamento de Energía de los EEUU, por el National Renewable Energy Laboratory and Conserval Engineering Inc., una compañía privada de calefacción solar y de conservación de energía.

La Fachada Ventilada Activa -FVA- de este proyecto se define como un muro parietodinámico con hoja exterior opaca (8). En un muro parietodinámico, el aire de ventilación es obtenido del exterior mediante ventilación forzada, precalentado en el interior de la cámara e introducido al edificio cuando existe necesidad de calefacción. Cuando el edificio no necesita ser calefactado, el aire de la cámara es liberado directamente al exterior (Figura 1).

El primer prototipo (Figura 1), construido con una hoja exterior de $2 \mathrm{~mm}$ de acero galvanizado y una cámara de aire de $3 \mathrm{~cm}$, fue ensayado en los Laboratorios de Control de Calidad del Gobierno Vasco siguiendo el procedimiento Paslink.

La utilización de este tipo de tecnologías para el ahorro de energía (9) es particularmente necesaria en España donde la transposición de la Directiva 2002/91/EC sobre la eficiencia energética de los edificios no entró en vigor hasta el 2006 con el CTE. Este hecho ha originado, consecuentemente, la construcción de muchos edificios derrochadores y en particular, el incremento del número de edificios de oficinas, por lo que constituye un factor de peso a considerar en la evaluación de la conveniencia de la FVA.

Con estas consideraciones, el Gobierno de España ha promovido y financiado numerosos proyectos e investigaciones con la intención de buscar alternativas al modo de construcción tradicional. 
Para evaluar la validez, en términos de ahorro de energía (10), de la FVA aplicada a edificios de oficinas en España ha sido necesario determinar y cuantificar el consumo de edificios de oficinas típicos que cumplen con lo establecido en el CTE. Para ello se definieron y simularon ocho edificios de oficinas tipo utilizando el programa $L I D E R$, el método general reconocido en el $C T E$ que determina la demanda energética de edificios.

La sencillez de la tecnología utilizada en la FVA, permite que la solución sea muy atractiva para ser aplicada tanto en edificios nuevos como en rehabilitación. La determinación del ahorro de energía que presenta la FVA ha sido obtenido utilizando esta herramienta gubernamental. Para poder simular un elemento especial como es la FVA en LIDER se ha desarrollado una aplicación específica en el programa y su integración en la versión oficial se encuentra en fase de estudio (11).

Finalmente, los resultados obtenidos tras las simulaciones han sido recogidos en forma de fichas y constituyen, por sí solos, una herramienta que permite evaluar el uso de la FVA y el ahorro energético teórico que se puede obtener tras su aplicación.

\section{OBJETIVOS}

Europa se enfrenta a un cambio radical en términos de eficiencia energética de los edificios. La construcción de edificios con muy bajo, casi cero, consumo energético es uno de los objetivos más ambiciosos establecidos en la recientemente aprobada Directiva 2010/31/UE. Por esta razón, los objetivos establecidos en la anterior Directiva 2002/91/EC y su transposición en el reglamento español quedan obsoletos.

Por otro lado, la muy tardía entrada en vigor del CTE (Marzo 2006) fue acompañada de una frenética actividad constructiva en España (Tabla 1). Ello conlleva que la mayoría de edificios construidos actualmente en España no cumplen con los mínimos establecidos en el CTE: esto significa que en un periodo de tiempo no muy lejano, muchos de los edificios construidos recientemente deberán ser rehabilitados energéticamente para poder cumplir los nuevos requisitos Europeos. Más aún, la inminente entrada en vigor del proceso de certificación energética de edificios existentes acentuará aún más la necesidad de actuar sobre ellos.

En este escenario debe considerarse además, la peculiaridad de la economía española. Con la deslocalización de la industria española hacia otros países, está emergiendo una nueva tipología edificatoria con la sustitución de plantas industriales por edificios de oficinas (12).

Desde 1990 el consumo de energía de los edificios de oficinas ha aumentado un $300 \%$ y es el responsable del $47,48 \%$ del consumo de energía en el sector servicios según datos del 2008 (el 6\% del total de consumo de energía en España) (13).

El impacto energético de este sector se prevé irá en aumento considerando la proliferación, en la última década, de edificios de cristal altamente derrochadores donde los conceptos relacionados con la modernidad, la tecnología y la transparencia predominan en su diseño (14).

Los edificios de oficinas van a pasar a ser una de las tipologías edificatorias más importantes de la siguiente década en España pero necesitan, del mismo modo, ser rediseñados y adaptados al nuevo marco energético. Con este claro objetivo, la fachada no puede desempeñar sólo un papel decorativo. Con todas estas consideraciones queda claro que la integración de sistemas industrializados de ahorro de energía integrados en la envolvente de los edificios (15), como la FVA, vayan proliferando en el futuro más cercano.

El objetivo principal de este trabajo ha sido estimar la eficiencia energética de la FVA, para reducir la demanda de calefacción, aplicada a edificios de oficinas en España y determinar la validez del sistema para poder alcanzar los nuevos objetivos marcados en la Directiva 2010/31/EU. El tipo de FVA utilizado en este análisis ha sido el primer prototipo desarrollado en el proyecto coordinado "Fachadas Ventiladas activas" ya descrito con anterioridad.

Se realizó un estudio para determinar "las características típicas de los edificios de oficinas en España". Este análisis posibilitó la definición de una serie de edificios tipo que representan las características más típicas de las oficinas (16) (17) (18).

Tabla 1. Número de visados de dirección de obra durante 2000 - 2009 clasificados por tipo de obra registrados en los Colegios de Arquitectos de España (Ministerio de Fomento 2009).

\begin{tabular}{|c|c|c|c|}
\hline Año & $\begin{array}{c}\text { Rehabilitación } \\
\text { o ampliación }\end{array}$ & Nueva planta & $\begin{array}{c}\text { Visados de dirección } \\
\text { de obra }\end{array}$ \\
\hline 2000 & 23240 & 89889 & 143902 \\
\hline 2001 & 25952 & 82803 & 140618 \\
\hline 2002 & 24977 & 82569 & 136544 \\
\hline 2003 & 26793 & 94476 & 152785 \\
\hline 2004 & 29335 & 102121 & 166437 \\
\hline 2005 & 33183 & 107577 & 179257 \\
\hline 2006 & 32051 & $\mathbf{1 1 3 0 4 1}$ & 181702 \\
\hline 2007 & 35610 & 86357 & 151730 \\
\hline 2008 & 34679 & 50959 & 839108 \\
\hline 2009 & 36947 & 31600 & \\
\hline
\end{tabular}


2. Esquema del principio de equivalencia
Posteriormente, se utilizó el programa $L /$ $D E R$ para obtener "edificios de referencia" para cada una de las zonas climáticas. Se obtuvieron un total de 192 casos de estudio.

Finalmente, los 192 edificios de referencia fueron utilizados para determinar la eficiencia energética de la FVA aplicada en la parte opaca de la fachada sur de cada caso. Con estos 192 casos de estudio no se pretende simplificar la complejidad y naturaleza específica de los proyectos reales, para ello sería necesario realizar un análisis más detallado. El objetivo es, por tanto, proporcionar una aproximación inicial cuantificada que refleje la influencia de ciertos parámetros en el comportamiento energético de los edificios de oficinas en España, con y sin el uso de una FVA.

\section{PRINCIPIO DE EQUIVALENCIA}

LIDER es el método general de cálculo de la demanda energética de los edificios reconocido por el CTE. La nueva versión ofrecerá la posibilidad de incorporar elementos especiales como es la FVA para el cálculo de la demanda energética.

Para poder integrar la FVA en LIDER se ha utilizado el principio de equivalencia que consiste en encontrar uno o una suma de elementos convencionales (muros y ventanas) que presentan un comportamiento igual o muy parecido al del elemento especial. A dicho o dichos elementos convencionales se les denomina "elementos equivalentes". Para que los elementos equivalentes puedan reproducir o aproximarse al comportamiento de los elementos

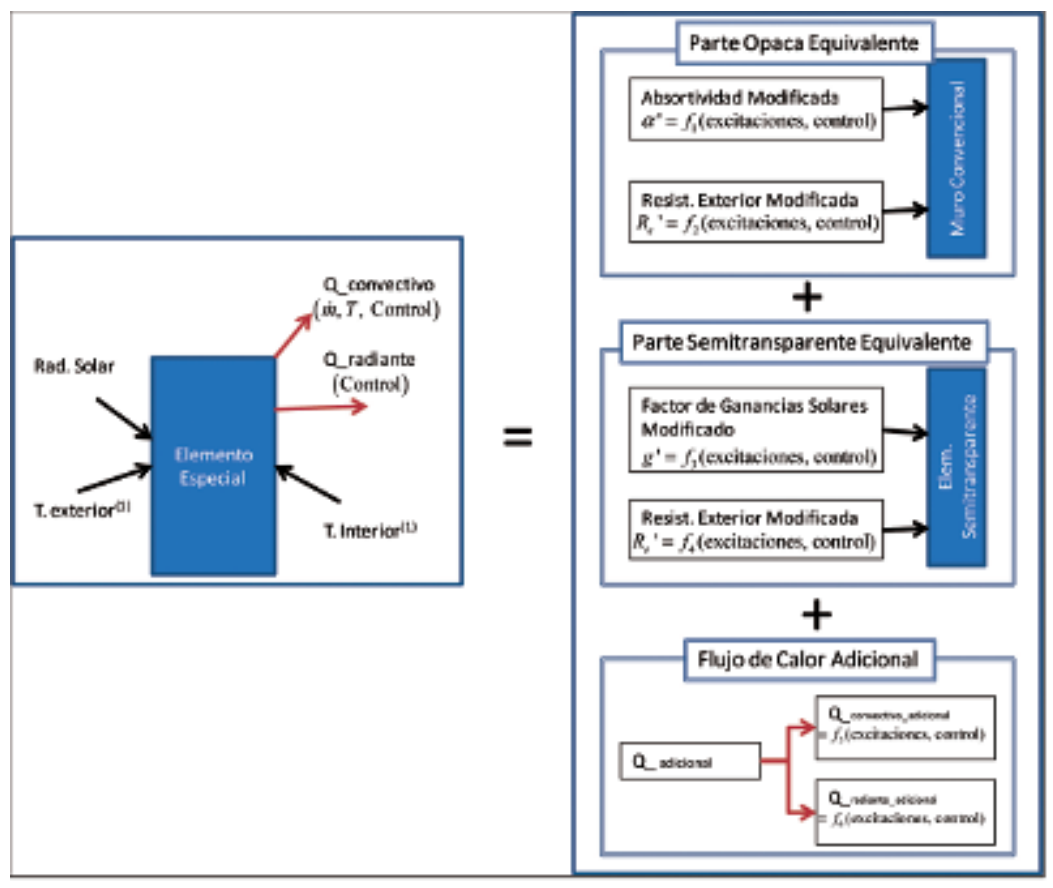

especiales es necesario que algunas de sus propiedades difieran de las del estándar de cálculo del programa $L I D E R$ y que además puedan ser variables en el tiempo.

Las propiedades del elemento convencional sobre las que se realizan modificaciones son aquellas relacionas con las excitaciones a las que es sometido, siendo esta, de dos tipos: Excitaciones de Temperatura ${ }^{1}$ y Excitaciones de Radiación Solar, y por tanto las propiedades sobre las que se realizan modificaciones son los coeficientes, convectivo y radiante, las absortividades solares y el factor de ganancias solares.

Con carácter general, lo que se postula con el principio de equivalencia, es que un elemento especial puede ser representado como la suma de un muro convencional, una ventana convencional y un flujo de calor aplicado al interior del edificio, esto es:

La respuesta térmica de un elemento especial de fachada ante las excitaciones de radiación y temperaturas exteriores e interiores, es igual a la suma de las respuestas de:

- Un muro convencional con resistencias convectivo-radiantes exterior e interior modificadas y una absortividad solar modificada. Pudiendo ser estas resistencias y absortividad, variables en el tiempo y dependientes de las excitaciones exteriores, interiores y de unas leyes de control.

- Una ventana convencional (elemento semitransparente) con resistencias convectivo-radiantes exterior e interior modificadas y un factor de ganancias solares modificado. Pudiendo ser dichos parámetros, variables en el tiempo y dependientes de las excitaciones exteriores, interiores y de unas leyes de control.

- Flujo de calor adicional que puede tener una parte convectiva y otra radiante, pudiendo ser variables en el tiempo y dependientes de las excitaciones exteriores, interiores y de unas leyes de control.

En la Figura 2 se muestra el esquema general del principio de equivalencia.

\section{METODOLOGÍA}

Para determinar las características típicas de los edificios de oficinas en España e identificar las tipologías más comunes, se han analizado revistas de arquitectura de carácter generalista de mayor difusión en España (19) (20) (21). Los edificios publicados en ellas reflejan el "estilo" de la época y establecen las tendencias respecto a materiales, tipologías, etc, que los arquitectos tienden a trasladar a sus proyectos. Estas publicaciones proporcionan, por tanto, una visión 
amplia del escenario construido en España, ya que representan los modelos en los que la mayoría de los edificios construidos en España están basados.

El periodo de consulta ha sido de 2000 a 2010. El año de referencia para el estudio fue establecido en 2002, año en que se publicó la Directiva 2002/91/EC, considerando que dicho acontecimiento marcó un antes y un después con respecto al comportamiento energético de los edificios, se presumía además que dicho evento había tenido su reflejo en las publicaciones de esos años. La consulta fue iniciada desde 2000, con el propósito de incluir el escenario previo a tal importante hecho.

Los parámetros para definir los edificios tipo han sido:

- Tipologías de edificios de oficinas más habituales (Figura 3):

- Edificio tipo U

- Edificio en torre con núcleo de comunicaciones en fachada

- Edificio en torre con núcleo de comunicaciones central

- Edificio tipo anillo

- Edificio tipo L

- Edificio tipo lineal

- Edificio tipo disperso

- Edificio tipo compacto

- Porcentaje típico de acristalamiento en fachada:

- $30 \%$ de acristalamiento en fachada

- $60 \%$ de acristalamiento en fachada

(El caso de $100 \%$ de acristalamiento en fachada no ha sido considerado en este estudio ya que no permite la aplicación de la $F V A$, elemento opaco por naturaleza).
Ocho tipologías diferentes y dos porcentajes de acristalamiento en fachadas definen un total de 16 edificios tipo y forman la base fundamental de este estudio. Los 16 edificios fueron posteriormente modelizados utilizando LIDER para cada una de las 12 zonas climáticas del CTE. Estos 192 modelos han generado otros tantos "edificios de referencia" con LIDER.

El cálculo de la demanda energética en LIDER se realiza mediante una simulación multizona transitoria, que también considera los puentes térmicos. El programa ha sido específicamente diseñado para la normativa española y realiza una comparación entre el edificio de referencia y el edificio objeto de estudio.

El edificio de referencia es idéntico al edificio real, con la excepción de su envolvente que cumple con los mínimos requisitos establecidos en la normativa. Los mínimos varían según la zona climática en la que se localiza el edificio, la orientación y el porcentaje de acristalamiento en la fachada. Por ejemplo en la zona climática más fría, zona E1, el valor de transmitancia térmica $(U)$ permitido para los cerramientos es de $0,57 \mathrm{~W} / \mathrm{m}^{2} \mathrm{~K}$ mientras que en la zona climática más cálida, zona A4, es de $0,94 \mathrm{~W} / \mathrm{m}^{2} \mathrm{~K}$.

El valor $U$ para las ventanas es más bajo en climas más fríos y en fachadas con una mayor superficie acristalada. Por ejemplo: en la zona E1, en una fachada orientada al norte y un $55 \%$ de acristalamiento en fachada el máximo valor $U$ permitido es de $1,9 \mathrm{~W} / \mathrm{m}^{2} \mathrm{~K}$, mientras que en la zona $\mathrm{A} 4$, en una fachada con orientación sur y un $20 \%$ de acristalamiento es de 5,7 W/m² $\mathrm{K}$.

Las tablas completas se encuentran en el Documento Básico HE1 - del CTE.

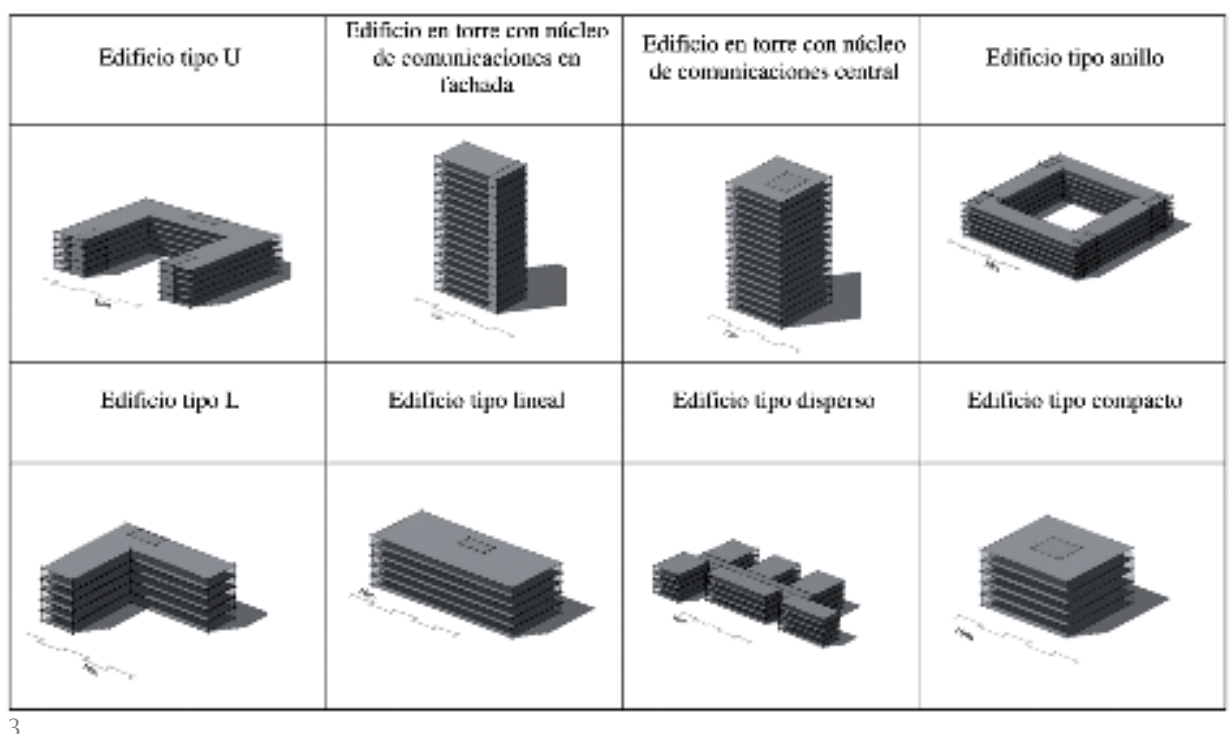




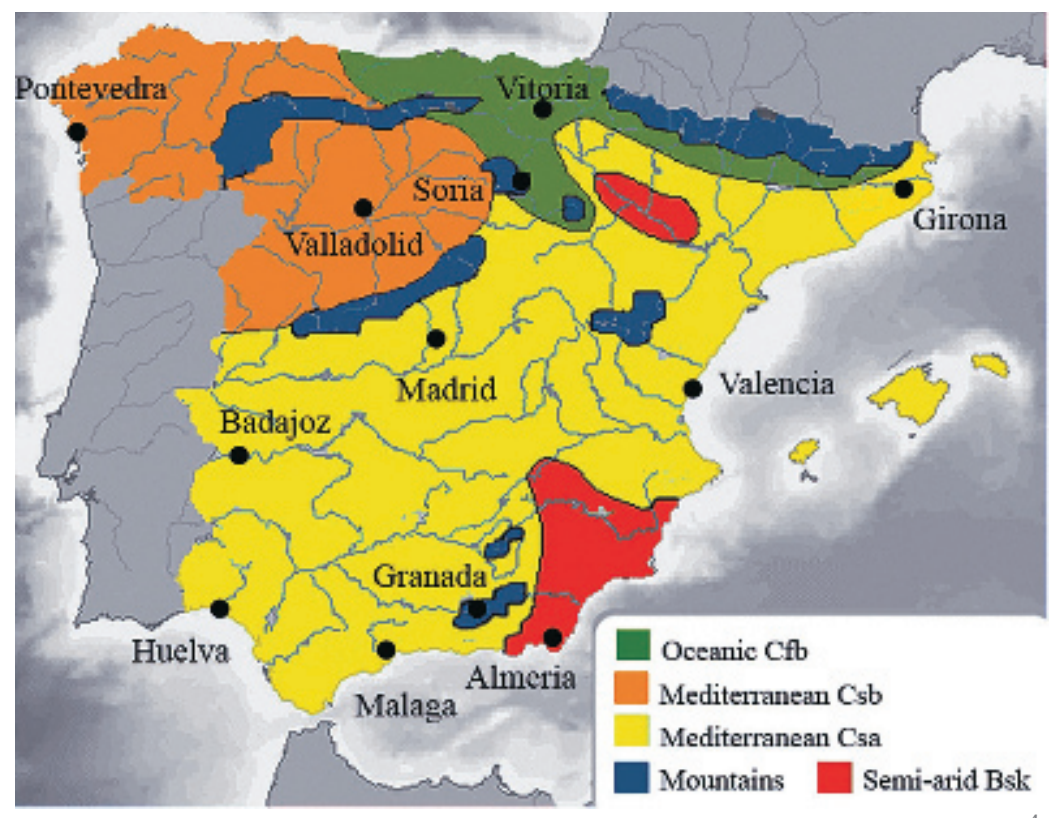

4. Mapa de clasificación climática de Köppen-Geiger para España

Cfb: templado cálido/ muy húmedo/ verano cálido

Csb: templado cálido/ verano seco/ verano cálido

Csa: templado cálido/ verano seco/ verano muy cálido

Bsk: árido/verano seco/frío árido.
El horario de funcionamiento establecido para los edificios simulados es 07:00 a 15:00 y de 17:00 a 20:00 durante la semana y de 07:00 a 15:00 los sábados. Las cargas internas en ese horario son:

- Personas: $10 \mathrm{~W} / \mathrm{m}^{2}$

- Iluminación: $7,5 \mathrm{~W} / \mathrm{m}^{2}$

- Equipos: $7,5 \mathrm{~W} / \mathrm{m}^{2}$

(La renovación de aire, considerando también las infiltraciones, es de 1 renovación por hora durante las 24 horas del día).

Para garantizar que los edificios de referencia estudiados sean lo más eficientes posibles, los edificios han sido estratégicamente orientados para adaptarse a las condiciones climáticas y geográficas específicas (Tabla 2).

La orientación óptima es aquella que posibilita recibir la máxima radiación solar en invierno y la mínima en verano. El cálculo de la orientación óptima fue realizado con el programa Weather ToolTM 2.00 (22). La orientación óptima no coincide exactamente con el ángulo de mayor radiación solar en invierno, sino que aparece ligeramente girado hacia el este para evitar el calor de la tardes de verano.

Las 12 zonas climáticas que se mencionan en el CTE (23) están definidas en base a la severidad climática de invierno y de verano, para determinadas localidades españolas.

La severidad climática combina los gradosdía y la radiación solar de una localidad, de manera que es posible demostrar que cuando dos localidades presentan la misma severidad climática en invierno $(\mathrm{SCl})$, la demanda energética de calefacción de un mismo edificio localizado en cada una de dichas localidades, es básicamente similar. Esto puede aplicarse también a la severidad climática en verano (SCV).

En este trabajo, por razones prácticas, se ha considerado una única ciudad como representativa de cada zona climática (Figura 4). A la hora de seleccionar una ciudad representativa para una zona climática, se ha calculado la media aritmética de las latitudes; aquella ciudad que más se aproxime a dicho valor ha sido considerada como ciudad representativa.

A partir de los 192 edificios estratégicamente orientados, se ha calculado su demanda de calefacción. La estimación de la eficiencia energética de la FVA está basada en el concepto de edificio de referencia mencionado con anterioridad.

Con tal fin, las partes opacas de las fachadas sur de estos edificios fueron modificadas mediante la superposición de FVAs. Estos edificios modificados fueron

Tabla 2. Orientación óptima para las ciudades representativas en cada zona climática. Radiación media diaria incidente en superficie vertical. Ángulo de la orientación óptima $\left(^{\circ}\right)$ medido en sentido horario desde el sur.

\begin{tabular}{|c|c|c|c|c|}
\hline $\begin{array}{c}\text { ZONA } \\
\text { CLIMÁTICA }\end{array}$ & $\begin{array}{c}\text { CIUDAD } \\
\text { REPRESENTATIVA }\end{array}$ & $\begin{array}{c}\text { ORIENTACIÓN } \\
\left.\text { ÓPTIMA } \mathbf{(}^{\circ}\right)\end{array}$ & $\begin{array}{c}\text { RADIACIÓN MEDIA } \\
\text { DIARIA EN INVIERNO } \\
{\left[\mathbf{k W h} / \mathbf{m}^{2}\right]}\end{array}$ & $\begin{array}{c}\text { RADIACIÓN MEDIA } \\
\text { DIARIA EN VERANO } \\
{\left[\mathbf{k W h} / \mathbf{m}^{2}\right]}\end{array}$ \\
\hline A3 & Málaga & $+5,0$ & 2,68 & 1,59 \\
\hline A4 & Almería & $+7,5$ & 2,91 & 1,59 \\
\hline B3 & Valencia & $+10,0$ & 2,54 & 1,66 \\
\hline B4 & Huelva & $+12,5$ & 2,29 & 1,76 \\
\hline C1 & Pontevedra & $-10,0$ & 1,57 & 1,49 \\
\hline C2 & Gerona & 0,0 & 2,33 & 1,45 \\
\hline C3 & Granada & $+2,5$ & 2,64 & 1,23 \\
\hline C4 & Badajoz & $+2,5$ & 2,08 & 1,47 \\
\hline D1 & Vitoria & $-10,0$ & 1,31 & 1,96 \\
\hline D2 & Valladolid & 0,0 & 1,52 & 1,35 \\
\hline D3 & Madrid & $-5,0$ & 1,98 & 1,88 \\
\hline E1 & Soria & $-5,0$ & 1,79 & \\
\hline
\end{tabular}




\begin{tabular}{|c|c|c|c|c|c|c|c|c|c|c|c|c|c|}
\hline \multirow[b]{4}{*}{ Edificio tipo $U$} & \multirow{2}{*}{$\begin{array}{l}\text { Superficie } \\
\text { acristalada } \\
\text { en fachada }\end{array}$} & \multicolumn{12}{|c|}{ Zonas climáticas del CTE } \\
\hline & & $\mathrm{A} 3$ & $\mathrm{~A} 4$ & $\mathrm{~B} 3$ & $\mathrm{~B} 4$ & $\mathrm{C} 1$ & $\mathrm{C} 2$ & C3 & $\mathrm{C} 4$ & D1 & $\mathrm{D} 2$ & D3 & E1 \\
\hline & $30 \%$ & & & & & & & & & & & & \\
\hline & $60 \%$ & & & & & & & & & & & & \\
\hline \multirow{2}{*}{$\begin{array}{l}\text { Edificio en torre con núcleo de } \\
\text { comunicaciones en fachada }\end{array}$} & $30 \%$ & & & & & & & & & & & & \\
\hline & $60 \%$ & & & & & & & & & & & & \\
\hline \multirow{2}{*}{$\begin{array}{l}\text { Edificio en torre con núcleo de } \\
\text { comunicaciones central }\end{array}$} & $30 \%$ & & & & & & & & & & & & \\
\hline & $60 \%$ & & & & & & & & & & & & \\
\hline \multirow[b]{2}{*}{ Edificio tipo anillo } & $30 \%$ & & & & & & & & & & & & \\
\hline & $60 \%$ & & & & & & & & & & & & \\
\hline \multirow[b]{2}{*}{ Edificio tipo $L$} & $30 \%$ & & & & & & & & & & & & \\
\hline & $60 \%$ & & & & & & & & & & & & \\
\hline \multirow[b]{2}{*}{ Edificio tipo lineal } & $30 \%$ & & & & & & & & & & & & \\
\hline & $60 \%$ & & & & & & & & & & & & \\
\hline \multirow[b]{2}{*}{ Edificio tipo disperso } & $30 \%$ & & & & & & & & & & & & \\
\hline & $60 \%$ & & & & & & & & & & & & \\
\hline \multirow[b]{2}{*}{ Edificio tipo compacto } & $30 \%$ & & & & & & & & & & & & \\
\hline & $60 \%$ & & & & & & & & & & & & \\
\hline
\end{tabular}

\footnotetext{
$\square$ Demanda de calefacción $<30 \mathrm{kwh} / \mathrm{m}^{2} \mathrm{a}$

Demanda de calefacción entre 30-60 kwh/m²a
}

\section{Demanda de calefacción entre $60-90 \mathrm{kwh} / \mathrm{m}^{2} \mathrm{a}$}

Demanda de calefacción entre $90-110 \mathrm{kwh} / \mathrm{m}^{2} \mathrm{a}$ posteriormente simulados con el programa LIDER. Para concluir, los resultados se presentan y comparan con los valores de sus correspondientes edificios de referencia. De esta manera, es posible determinar el ahorro energético que ofrece la FVA para cada uno de los casos.

\section{DEMANDA DE CALEFACCIÓN DE EDIFICIOS DE REFERENCIA}

El cálculo de la demanda de calefacción de los edificios de referencia ha sido realizado considerando diferentes parámetros: la tipología, el porcentaje de acristalamiento en fachada y la zona climática. Tras realizar un análisis individual de cada una de las tipologías, se han obtenido una serie de conclusiones. Los valores máximos y mínimos para cada una de las tipologías quedan recogidos en la Tabla 3.

Según los casos estudiados, se obtiene que la demanda de calefacción es mayor en los casos con un $30 \%$ de acristalamiento en fachada que en los casos con un $60 \%$. Éste hecho es debido a que cuanto mayor sea la superficie acristalada, mayor es el aprovechamiento de la energía solar. Por otro lado, hay que subrayar, que las propiedades de las ventanas varían dependiendo de la superficie acristalada dado que el CTE exige una menor transmitancia térmica para mayores superficies acristaladas.

Por otro lado, se observa una diferencia notable entre la demanda de calefacción en los climas más fríos (D2, D1 y E1) y los más calurosos (A3, A4 y B4). Los valores varían entre un máximo de $79,62 \mathrm{kWh} / \mathrm{m}^{2}$ a y un mínimo de $0,17 \mathrm{kWh} / \mathrm{m}^{2} \mathrm{a}$.
En general, el factor de forma (superficie de envolvente/volumen) tienen un gran impacto en la demanda de calefacción: cuanto mayor sea el factor de forma (mayor superficie para un mismo volumen), mayor es la demanda.

Por lo tanto, la demanda de calefacción depende de multitud de factores: la superficie acristalada, las características del vidrio, el factor de forma, la tipología y la zona climática.

En la Figura 5 se muestra a modo de resumen, el rango de valores de la demanda de calefacción en base a la superficie acristalada, tipología y zona climática. De la tabla se obtiene que la demanda de calefacción, en la mayoría de los casos, es inferior a $30 \mathrm{kWh} / \mathrm{m}^{2} \mathrm{a}$. La demanda de calefacción es, obviamente, mayor en las zonas climáticas más frías donde se alcanzan valores entre 30 - $60 \mathrm{kWh} / \mathrm{m}^{2}$ a y $60-90 \mathrm{kWh} / \mathrm{m}^{2} \mathrm{a}$.

Por tanto, el CTE permite la construcción de edificios con una elevada demanda de

Tabla 3. Demanda máxima y mínima de calefacción de los edificios de referencia por tipologías

\begin{tabular}{|c|c|c|c|c|}
\hline TIPOLOGÍA & $\begin{array}{c}\text { MÁXIMO } \\
{\left[\mathbf{k W h} / \mathbf{m}^{2} \mathbf{a}\right]}\end{array}$ & $\begin{array}{c}\text { ZONA } \\
\text { CLIMÁTICA }\end{array}$ & $\begin{array}{c}\text { MíNIMO } \\
{\left[\mathbf{k W h} / \mathbf{m}^{2} \mathbf{a}\right]}\end{array}$ & $\begin{array}{c}\text { ZONA } \\
\text { CLIMÁTICA }\end{array}$ \\
\hline Edificio tipo U & 77,29 & Soria (E1) & 2,84 & Almería (A4) \\
\hline Torre $1^{(*)}$ & 57,13 & Soria (E1) & 0,17 & Almería (A4) \\
\hline Torre $2^{(* *)}$ & 50,09 & Soria (E1) & 1,23 & Almería (A4) \\
\hline Edificio tipo anillo & 79,62 & Soria (E1) & 4,28 & Almería (A4) \\
\hline Edificio tipo L & 66,71 & Soria (E1) & 1,62 & Almería (A4) \\
\hline Edificio tipo lineal & 54,36 & Soria (E1) & 1,84 & Almería (A4) \\
\hline Edificio tipo disperso & 71,94 & Soria (E1) & 4,60 & Almería (A4) \\
\hline Edificio tipo compacto & 60,78 & Soria (E1) & 2,10 & Almería (A4) \\
\hline
\end{tabular}

Torre $1^{(*)}$ : Edificio en torre con núcleo de comunicaciones en fachada

Torre $2^{(* *)}$ : Edificio en torre con núcleo de comunicaciones central 
6. Disminución de la demanda de calefacción utilizando tecnología FVA respecto a los valores de referencia (cumplimiento de CTE). calefacción. Este hecho contradice el objetivo establecido por la nueva directiva en la que se pretenden conseguir edificios con un consumo de energía casi nulo.

\section{DEMANDA DE CALEFACCIÓN DE EDIFICIOS CON FVA}

Para estimar la eficiencia energética de la FVA se han considerado como base los valores obtenidos en los 192 edificios de referencia mencionados anteriormente. La superficie opaca de las fachadas con orientación sur de cada uno de los edificios, ha sido modificada mediante la adición de una FVA. Estos edificios han sido simulados mediante LIDER para obtener su demanda energética. Los resultados se presentan en comparación con los valores de sus correspondientes edificios de referencia. De esta manera, ha sido posible establecer el ahorro energético, en términos de reducción de la demanda de calefacción, que ofrece una FVA en cada uno de los casos estudiados.

\subsection{Demanda de calefacción para edificios con un $30 \%$ de superficie acristalada}

En general, se concluye que la utilización de FVAs en edificios con un $30 \%$ de acristalamiento en fachada mejora su eficiencia energética en comparación con los valores de referencia. Este hecho se confirma para todas las zonas climáticas. El comportamiento de la FVA es óptimo en zonas climáticas más templadas. En la siguiente tabla (Tabla 4) se muestra que en la mayo- ría de los casos estudiados se alcanza un ahorro superior al $20 \%$.

Analizando los resultados por tipologías, en el edificio tipo $L$ se obtienen los mejoras datos. En todas las zonas climáticas, a excepción de D1, D2 y E1, se alcanzan ahorros superiores al 40\% (Figura 6).

El edificio torre con núcleo de comunicaciones en fachada, el edificio tipo $L$ y el edificio tipo compacto, presentan ahorros energéticos similares. En estos casos se supera el $40 \%$ de ahorro en las zonas climáticas A3, A4, B3 y B4 y entre el 20$40 \%$ en muchas otras zonas. Por otro lado, en las zonas climáticas con inviernos muy fríos (D1 y E1) el ahorro obtenido es en torno al $10 \%$.

\subsection{Demanda de calefacción para edificios con un $60 \%$ de superficie acristalada}

En general, se concluye que la utilización de FVAs en edificios con un $60 \%$ de acristalamiento en fachada mejora su eficiencia energética en comparación con los valores de referencia. Este hecho se confirma para todas las zonas climáticas. El comportamiento de la FVA es óptimo en zonas climáticas más templadas.

Sin embargo, en comparación con los resultados obtenidos con un $30 \%$ de acristalamiento en fachada, la mejora que aporta la FVA no es tan evidente. Esto hecho es debido a que con una mayor superficie acristalada la superficie disponible para la FVA es inferior.

\begin{tabular}{|c|c|c|c|c|c|c|c|c|c|c|c|c|c|}
\hline \multirow[b]{4}{*}{ Edificio tipo $U$} & \multirow{2}{*}{$\begin{array}{l}\text { Superficie } \\
\text { acristalada } \\
\text { en fachada }\end{array}$} & \multicolumn{12}{|c|}{ Zonas climáticas del CTE } \\
\hline & & A3 & A4 & B3 & B4 & C1 & $\mathrm{C} 2$ & $\mathrm{C} 3$ & $\mathrm{C} 4$ & D1 & $\mathrm{D} 2$ & D3 & E1 \\
\hline & $30 \%$ & & & & & & & & & & & & \\
\hline & $60 \%$ & & & & & & & & & & & & \\
\hline \multirow{2}{*}{$\begin{array}{l}\text { Edificio en torre con núcleo de } \\
\text { comunicaciones en fachada }\end{array}$} & $30 \%$ & & & & & & & & & & & & \\
\hline & $60 \%$ & & & & & & & & & & & & \\
\hline \multirow{2}{*}{$\begin{array}{l}\text { Edificio en torre con núcleo de } \\
\text { comunicaciones central }\end{array}$} & $30 \%$ & & & & & & & & & & & & \\
\hline & $60 \%$ & & & & & & & & & & & & \\
\hline \multirow[b]{2}{*}{ Edificio tipo anillo } & $30 \%$ & & & & & & & & & & & & \\
\hline & $60 \%$ & & & & & & & & & & & & \\
\hline \multirow[b]{2}{*}{ Edificio tipo $L$} & $30 \%$ & & & & & & & & & & & & \\
\hline & $60 \%$ & & & & & & & & & & & & \\
\hline \multirow[b]{2}{*}{ Edificio tipo lineal } & $30 \%$ & & & & & & & & & & & & \\
\hline & $60 \%$ & & & & & & & & & & & & \\
\hline \multirow[b]{2}{*}{ Edificio tipo disperso } & $30 \%$ & & & & & & & & & & & & \\
\hline & $60 \%$ & & & & & & & & & & & & \\
\hline \multirow[b]{2}{*}{ Edificio tipo compacto } & $30 \%$ & & & & & & & & & & & & \\
\hline & $60 \%$ & & & & & & & & & & & & \\
\hline
\end{tabular}


Tabla 4. Demanda de calefacción $\left[\mathrm{kWh} / \mathrm{m}^{2} \mathrm{a}\right]$ para edificios con $F V A$ y una superficie acristalada en fachada del $30 \%$ con respecto a los valores de referencia

\begin{tabular}{|c|c|c|c|c|c|c|c|c|c|c|c|c|c|}
\hline & & A3 & A4 & B3 & B4 & C1 & $\mathrm{C} 2$ & C3 & $\mathrm{C} 4$ & D1 & D2 & D3 & E1 \\
\hline \multirow{2}{*}{ EDIFICIO TIPO U } & $F V A$ & 5,46 & 2,91 & 12,13 & 6,67 & 71,15 & 37,01 & 31,25 & 21,28 & 63,18 & 64,72 & 38,63 & 71,11 \\
\hline & REF & 10,35 & 8,01 & 19,80 & 10,54 & 77,29 & 45,00 & 37,97 & 28,52 & 72,20 & 65,14 & 45,71 & 77,29 \\
\hline \multirow{2}{*}{ TORRE 1 (*) } & $F V A$ & 1,04 & 0,81 & 6,42 & 1,90 & 10,23 & 23,94 & 1,90 & 12,46 & 41,58 & 0,00 & 10,23 & 50,12 \\
\hline & $R E F$ & 5,22 & 2,63 & 12,09 & 6,52 & 17,94 & 31,49 & 6,52 & 19,17 & 47,42 & 0,17 & 17,94 & 57,13 \\
\hline \multirow{2}{*}{ TORRE 2(**) } & $F V A$ & 1,78 & 1,27 & 6,99 & 2,24 & 10,78 & 23,88 & 18,73 & 12,57 & 42,37 & 38,74 & 24,83 & 47,05 \\
\hline & REF & 3,68 & 1,72 & 9,84 & 5,44 & 15,85 & 28,40 & 22,67 & 16,20 & 46,55 & 42,36 & 28,31 & 50,09 \\
\hline \multirow{2}{*}{ EDIFICIO TIPO ANILLO } & $F V A$ & 8,31 & 6,17 & 16,47 & 8,20 & 22,41 & 39,06 & 33,03 & 24,00 & 62,66 & 57,47 & 39,61 & 76,88 \\
\hline & REF & 9,73 & 7,63 & 18,72 & 9,70 & 25,35 & 41,83 & 35,26 & 26,41 & 65,72 & 60,07 & 41,83 & 79,61 \\
\hline \multirow{2}{*}{ EDIFICIO TIPO L } & $F V A$ & 1,80 & 1,01 & 8,33 & 3,90 & 14,32 & 28,95 & 23,55 & 15,36 & 52,87 & 48,76 & 30,81 & 59,41 \\
\hline & REF & 7,37 & 5,22 & 14,52 & 7,58 & 21,91 & 37,00 & 30,40 & 22,64 & 62,55 & 55,55 & 37,73 & 66,70 \\
\hline \multirow{2}{*}{ EDIFICIO TIPO LINEAL } & $F V A$ & 0,75 & 0,11 & 8,37 & 1,29 & 6,77 & 17,92 & 14,60 & 9,08 & 49,82 & 45,51 & 19,39 & 56,45 \\
\hline & REF & 4,72 & 2,83 & 11,38 & 5,90 & 17,23 & 30,48 & 24,96 & 17,71 & 50,48 & 45,12 & 30,80 & 54,36 \\
\hline \multirow{2}{*}{ EDIFICIO TIPO DISPERSO } & $F V A$ & 7,39 & 5,66 & 14,95 & 7,39 & 21,11 & 37,19 & 33,64 & 22,26 & 60,76 & 55,94 & 37,96 & 66,81 \\
\hline & REF & 10,01 & 7,41 & 18,10 & 9,79 & 25,57 & 41,88 & 38,06 & 26,31 & 66,78 & 60,60 & 42,33 & 71,94 \\
\hline \multirow{2}{*}{ EDIFICIO TIPO СОМРАCTO } & FVA & 3,00 & 1,99 & 9,21 & 3,56 & 13,95 & 28,65 & 23,57 & 15,67 & 49,78 & 45,13 & 29,96 & 55,69 \\
\hline & REF & 6,80 & 4,24 & 13,89 & 7,25 & 20,28 & 34,75 & 28,55 & 21,07 & 56,32 & 50,53 & 35,00 & 60,78 \\
\hline
\end{tabular}

Torre $1\left(^{*}\right)$ : Edificio en torre con núcleo de comunicaciones en fachada

Torre $2\left({ }^{* *}\right)$ : Edificio en torre con núcleo de comunicaciones central

Tabla 5. Demanda de calefacción $\left[\mathrm{kWh} / \mathrm{m}^{2} \mathrm{a}\right]$ para edificios con $F V A$ y una superficie acristalada en fachada del $60 \%$ con respecto a los valores de referencia

\begin{tabular}{|c|c|c|c|c|c|c|c|c|c|c|c|c|c|}
\hline & & A3 & A4 & B3 & B4 & C1 & C2 & C3 & $\mathrm{C} 4$ & D1 & D2 & D3 & E1 \\
\hline \multirow{2}{*}{ EDIFICIO TIPO U } & $V A F$ & 2,14 & 0,56 & 54,96 & 2,14 & 14,80 & 29,06 & 23,32 & 16,55 & 54,96 & 51,09 & 31,73 & 62,37 \\
\hline & REF & 5,28 & 2,84 & 60,20 & 5,28 & 20,45 & 33,92 & 26,46 & 20,71 & 60,20 & 54,26 & 35,50 & 64,90 \\
\hline \multirow{2}{*}{ TORRE 1 (*) } & VAF & 0,00 & 0,00 & 3,25 & 0,27 & 7,24 & 17,65 & 12,86 & 9,14 & 39,79 & 36,42 & 19,33 & 3,45 \\
\hline & $R E F$ & 0,77 & 0,17 & 6,78 & 3,64 & 12,17 & 23,64 & 17,86 & 12,84 & 46,08 & 41,24 & 24,64 & 47,74 \\
\hline \multirow{2}{*}{ TORRE $2\left(^{* *}\right)$} & VAF & 1,40 & 1,02 & 4,50 & 1,59 & 9,64 & 20,83 & 15,83 & 11,28 & 40,91 & 36,65 & 22,19 & 45,04 \\
\hline & $R E F$ & 1,70 & 1,22 & 7,17 & 2,19 & 12,45 & 23,67 & 18,29 & 13,22 & 44,33 & 39,94 & 25,46 & 47,37 \\
\hline \multirow{2}{*}{ EDIFICIO TIPO ANILLO } & VAF & 5,37 & 3,64 & 12,90 & 5,37 & 19,98 & 34,48 & 27,89 & 20,56 & 59,74 & 54,29 & 35,79 & 64,88 \\
\hline & REF & 6,25 & 4,28 & 13,80 & 6,25 & 21,34 & 35,68 & 28,93 & 21,74 & 61,22 & 55,56 & 36,98 & 66,08 \\
\hline \multirow{2}{*}{ EDIFICIO TIPO L } & VAF & 1,20 & 0,67 & 7,64 & 2,71 & 13,81 & 26,01 & 21,03 & 14,81 & 51,60 & 47,26 & 13,81 & 55,90 \\
\hline & REF & 4,25 & 1,61 & 10,35 & 5,87 & 18,69 & 30,93 & 24,15 & 18,48 & 56,16 & 40,88 & 18,69 & 60,22 \\
\hline \multirow{2}{*}{ EDIFICIO TIPO LINEAL } & VAF & 0,93 & 0,04 & 6,46 & 2,28 & 12,38 & 23,75 & 18,55 & 12,89 & 15,69 & 40,99 & 25,69 & 49,15 \\
\hline & REF & 2,76 & 1,84 & 8,38 & 3,82 & 15,21 & 26,52 & 20,53 & 15,41 & 48,60 & 43,26 & 27,93 & 51,40 \\
\hline \multirow{2}{*}{ EDIFICIO TIPO DISPERSO } & VAF & 5,52 & 4,21 & 14,95 & 6,12 & 18,94 & 33,94 & 27,78 & 20,36 & 59,02 & 54,11 & 35,27 & 64,39 \\
\hline & REF & 6,70 & 4,60 & 18,10 & 7,64 & 22,03 & 36,75 & 29,95 & 22,42 & 62,37 & 56,52 & 37,73 & 66,99 \\
\hline \multirow{2}{*}{ EDIFICIO TIPO COMPACTO } & VAF & 2,17 & 1,62 & 7,78 & 2,25 & 12,85 & 25,66 & 20,01 & 14,14 & 48,94 & 44,01 & 27,80 & 53,99 \\
\hline & REF & 3,33 & 2,10 & 10,36 & 5,55 & 17,53 & 29,87 & 23,79 & 17,70 & 53,45 & 48,20 & 31,61 & 57,35 \\
\hline
\end{tabular}

Torre $1\left(^{*}\right)$ : Edificio en torre con núcleo de comunicaciones en fachada

Torre $2(* *)$ : Edificio en torre con núcleo de comunicaciones central

Analizando las diferentes tipologías, la FVA es más eficiente en la torre con el núcleo de comunicaciones en la fachada. Este hecho se confirma para todas las zonas climáticas, con la excepción de las zonas D1, D2, D3 y E1, donde se obtienen ahorros entre el $20-40 \%$ e incluso superando el $40 \%$ (Figura 6).

En los edificios tipo $L$ y $U$ se da un comportamiento energético similar de la FVA. En estos casos se obtiene un ahorro superior al $40 \%$ en las zonas A3, A4 y B4 y en torno al $20 \%$ en muchas otras zonas. En términos generales, en zonas climáticas con inviernos muy fríos (D1 y E1), el ahorro energético se obtiene con la aplicación de la FVA es en torno al $10 \%$.
En la Tabla 5 se muestra que en la mayoría de los casos estudiados se obtiene un ahorro energético en torno al $20 \%$.

\section{CONCLUSIONES}

El mero cumplimiento del CTE no garantiza la construcción de edificios energéticamente eficientes en España, más aún, dicha normativa permite la construcción de edificios de oficinas con una demanda energética superior a $100 \mathrm{kWh} / \mathrm{m}^{2} \mathrm{a}$.

Esto significa que la gran mayoría de edificios construidos en los últimos años en España necesitarán ser rehabilitados energéticamente para mejorar su comportamiento. 
En este artículo se ha analizado la eficacia de la FVA para reducir la demanda de calefacción, componente importante de la demanda energética total de un edificio de oficinas dependiendo de la zona climática en la que se encuentre.

Este valor varía entre un máximo de 79,69 $\mathrm{kWh} / \mathrm{m}^{2}$ a para un edificio tipo anillo situado en Soria (Zona climática E1) y un mínimo de $0,17 \mathrm{kWh} / \mathrm{m}^{2}$ a para un edificio en torre con núcleo de comunicaciones en fachada situado en Almería (zona climática A4).

Para todos los casos estudiados se concluye que la demanda de calefacción es mayor con un $30 \%$ que con un $60 \%$ de acristalamiento en fachada. Este hecho es debido a que la radiación incidente a través de las zonas acristaladas permite un mayor aprovechamiento de la energía solar.

Además hay que recalcar que dependiendo del porcentaje de vidrio en la fachada las propiedades del mismo varían: a mayor superficie acristalada el CTE exige una menor transmitancia térmica.

Tras las simulaciones llevadas a cabo utilizando el programa LIDER es posible establecer que la FVA presenta un comportamiento óptimo, permitiendo una reducción en la demanda de calefacción superior al $40 \%$ en muchos casos.

En zonas climáticas con inviernos suaves, por ejemplo, en un edificio tipo $U$ con un $30 \%$ de acristalamiento en fachada situado en la zona climática $\mathrm{A} 3$, la demanda de calefacción se reduce de 10,35 kWh/m²a a $5,46 \mathrm{kWh} / \mathrm{m}^{2} \mathrm{a}$.

En zonas climáticas templadas como son $\mathrm{C} 1, \mathrm{C} 2$ y $\mathrm{C} 3$, el potencial de ahorro medio es en torno al $10-20 \%$. Pero puede inclu- so mejorarse, por ejemplo, para un edificio tipo lineal con un $30 \%$ de acristalamiento en fachada situado en la zona climática C1 la demanda de calefacción se reduce de $17,23 \mathrm{kWh} / \mathrm{m}^{2} \mathrm{a}$ a $6,77 \mathrm{kWh} / \mathrm{m}^{2} \mathrm{a}$.

Finalmente, en zonas climáticas con inviernos muy fríos, la FVA permite lograr un ahorro en torno al $10 \%$. Pero puede incluso mejorarse, por ejemplo, para un edificio tipo lineal con un $30 \%$ de acristalamiento en fachada situado en la zona climática D3 la demanda de calefacción se reduce de $30,80 \mathrm{kWh} / \mathrm{m}^{2} \mathrm{a}$ a $19,39 \mathrm{kWh} / \mathrm{m}^{2} \mathrm{a}$.

En general, los edificios con un menor porcentaje de acristalamiento en fachada, es decir, con una mayor superficie de FVA presentan menores demandas energéticas.

La FVA, con un espesor inferior a los $5 \mathrm{~cm}$, es una interesante solución constructiva para la rehabilitación y construcción de edificios de oficinas en España.

\section{AGRADECIMIENTOS}

Ministerio de Educación y Ciencia; Escuela de Arquitectura de la Universidad del País Vasco (EHU/UPV); Departamento de Arquitectura de la Universidad del País Vasco (EHU/UPV); Group Building Environmental Studies de la Universidad de Atenas; Escuela Técnica Superior de Ingeniería de la Universidad del País Vasco (EHU/UPV); Departamento de Ingeniería Energética de la Universidad de Sevilla; Departamento de Máquinas y Motores Térmicos de la Universidad de Córdoba; Laboratorios de Control de Calidad de la Edificación del Gobierno Vasco; Mattheos Santamouris; Alfonso del Águila; Jose María Sala Lizarraga; José Luís Molina; Servando Állvarez; Manuel Ruiz de Adana; Aitor Erkorera; Iván Flores; Cesar Escudero; Christian Suárez y Fernando Peci.

\section{BIBLIOGRAFÍA}

(1) Irulegi, O.: Energy efficiency of Ventilated Active Façades applied to office buildings in Spain, PhD Thesis, Universidad del País Vasco EHU/UPV, 2011

(2) Arons, D.M.M.; Glicksman, L.R.: "Double Skin, Airflow Facades: will the Popular European Model work in the USA?". Proceedings of ICBEST 2001, International Conference on Building Envelope Systems and Technologies 1, pp 203- 207, Ottawa, 2001.

(3) Harrison, K.; Meyer-Boake, T.: The Tectonics of the Environmental Skin, University of Waterloo, School of Architecture, 2003. Disponible en http://www.fes.uwaterloo.ca/ architecture/faculty_projects/terri/ds/double.pdf

(4) Uuttu, S.: Study of Current Structures in Double-Skin Facades. MSc thesis in Structural Engineering and Building Physics, Department of Civil and Environmental Engineering, Helsinki University of Technology (HUT), 2001. Disponible en http://www.hut.fi/Units/ Civil/Steel/SINI2.PDF

(5) Saelens, D.: Energy Performance Assessments of Single Storey Multiple-Skin Facades. PhD thesis, Laboratory for Building Physics, Department of Civil Engineering, Catholic University of Leuven, 2002. Disponible en http://envelopes.cdi.harvard.edu/envelopes/ content/resources/pdf/case_studies/PhD_Dirk_Saelens.pdf 
(6) Belgian Building Research Institute - BBRI. Source book for a better understanding of conceptual and operational aspects of active facades, Bruselas, 2002. Disponible en http://www.bbri.be/activefacades/index2.htm

(7) Poirazis, H.: Single and double skin. Glazed office buildings. Analyses of energy use and indoor climate. PhD Thesis, Division of Energy and Building Design, Department of Architecture and Built Environment, Lund University, Faculty of Engineering, Lund, 2008.

(8) Ruiz-Pardo, A.: Ahorro energético mediante el uso de elementos de doble envolvente transparente-opaco. PhD Thesis, University of Seville, Sevilla, 2008.

(9) Commission of the European Communities. GREEN PAPER on Energy Efficiency - or Doing More With Less, Bruselas, 2005.

(10) Ebbert, T.: RE-FACE: Refurbishment Strategies for the Technical Improvement of Office Façades, PhD Thesis, Technische Universiteit Delft, Pubblished by Thiemo Ebbert, Bochum, 2010.

(11) Ruiz-Pardo, A.: "Simulation Model for Some Types of Double Envelope Elements". The International Journal of Ventilation 9 No 3, 2010.

(12) Ministerio de Industria, Turismo y Comercio. Gobierno de España. Globalización y deslocalización. Importancia y efectos para la industria española. División de Información, Documentación y Publicaciones, Centro de Publicaciones, Madrid, 2008.

(13) IDAE, Dpto. Planificación y Estudios, Segurado de Arriba, P. y García Montes, J.P. Evolución del consumo y de la intensidad energética en España, Análisis Global y Sectorial de la evolución del consumo y de la intensidad energética en España. Comparación a nivel europeo, 2008.

(14) Coyne, R.; Snodgrass, R.: "Metaphors in the design studio". Journal of Architectural Education 48 2, pp. 113-125, 1994.

(15) Orosa, J.A.; Oliveira, A.C.: "Energy saving with passive climate control methods in Spanish office buildings". Energy and Buildings 41, pp.823-828, 2009.

(16) Jpedicke, J.: Büro und Verwaltungsbauten. Internationale Beispiele. Informationsdaten in Bild und Text, Karl Krämer Verlag, Stuttgart, 1975.

(17) Manasseh, L.; Cunliffe. R.: Office Buildings. B.T. Bastford Ltd, Londres, 1962.

(18) Hascher, R.; Jeska, S.; Klauck, B.: Entwurf Bürobau, Bikhäuser- Verlag Architektur, Basilea/Berlin/Boston, 1985.

(19) Arquitectura Viva. nº70 -126, Arquitectura Viva SL, Madrid.

(20) Tectónica. nº 11-28, ATC Ediciones, Madrid.

(21) Detail. nº1(2002) - n. ${ }^{\circ} 1$ (2010), Institut für internationale Architektur-Dokumentation $\mathrm{GmbH}+\mathrm{Co} . \mathrm{KG}$, Múnich

(22) EnergyPlus Weather data on the website: http://apps1.eere.energy.gov

(23) Sanchez de la Flor, F.J.; Alvarez, S.; Molina, J.L.; Gonzalez, R.: "Climatic zoning and its application to Spanish building energy performance regulations". Energy and Buildings 40, pp.1984-1990, 2008. 